\title{
Molecular genetic diagnosis of Glanzmann syndrome in Iranian population; reporting novel and recurrent mutations
}

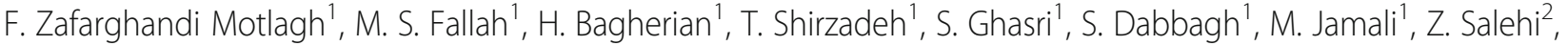 \\ M. Abiri ${ }^{1,3^{*}}$ and S. Zeinali ${ }^{1,4^{*}}$
}

\begin{abstract}
Background: Glanzmann thrombasthenia (GT) is a rare autosomal recessive abnormality of platelet aggregation with quantitative and/or qualitative abnormality of allb $\beta 3$ integrin. The allb $\beta 3$ is a platelet fibrinogen receptor, which is required for platelet aggregation, firm adhesion, and also spreading.

The disease is more prevalent in the populations with a higher rate of consanguineous marriages as in some Middle Eastern populations including Iraq, Jordan, and Iran. Different types of mutations in ITGA2B and ITGB3 genes have been previously reported to cause the disease.

Result: In this study, 16 patients with the clinical diagnosis of GT were studied. Direct sequencing of the exons and exon-intron boundaries of the above genes revealed mutations in 14 patients (detection rate: 87.5\%). Briefly, out of fifteen types of identified mutations, 14 were novel. Seven mutations in the ITGB3 gene included 4 missense [C.2T > C, c.155 G > T, c. 538 G > A, c.1990 G > T], one nonsense mutation [c.1303 G > T], a small deletion [c.1656_1658delCTC] and a deletion of one nucleotide [c.401delA]. Mutations in the ITGA2B were 8 different mutations consisting 2 missense [c.286 T > A, c.842 C > T, 2 deletions [c.1899 del T, c.189-319_236del], an insertion [c.1071_1072insG] and one splice site mutations [c.409-3 C > G], one synonymous mutation that might alter the normal splicing process [c.1392 A> G] and a nonsense mutation [c.1555 C > T].

The causative mutation in 2 patients remained unknown. Using long-range PCR and sequencing, we found a rather large deletion. The break point of this deletion covers $319 \mathrm{nt}$ from the last part of the first intron and $48 \mathrm{nt}$ from the beginning of the second exon of ITGA2B gene. The deletion was also detected in two unrelated patients with the same ethnicity. In addition, in silico analyses of novel mutations were performed.
\end{abstract}

Conclusion: There was no recurrent mutation in the studied population. This may be due to either small sample size or the heterogeneity of the studied population.

Keywords: Glanzmann thrombasthenia, $a_{\| b} \beta_{3}$, ITGA2B, ITGB3, Iran

\section{Introduction}

Glanzmann thrombasthenia (GT, OMIM: 273800) is a rare inherited disorder of platelet function, caused by quantitative or qualitative defects of the platelet membrane glycoprotein (GP) IIb-IIIa ( $\alpha$ IIb $\beta 3)$ complex [1]. Patients have lifelong mucosal bleeding and may require platelet transfusions for severe bleeding episodes. Bleeding

\footnotetext{
* Correspondence: abiri.m@iums.ac.ir; zeinali@gmail.com

'Dr. Zeinali's Medical Genetics Lab, Kawsar Human Genetics Research Center, No. 41 Majlesi St., Vali Asr St, Tehran 1595645513, Iran

Full list of author information is available at the end of the article
}

complications are also frequent after dental extraction, surgery, or delivery [2]. GT is a rare disorder worldwide but is relatively frequent in populations such as Iraqi, Jews, Jordanian Arabs, Iran, Palestine, South Indians and French Gypsies with a high frequency of consanguineous marriages $[3,4]$.

The molecular basis of GT is linked to quantitative and/ or qualitative abnormalities of $\alpha \operatorname{IIb} \beta 3$ integrin that mediates binding of the adhesive proteins to attach aggregating platelets and ensure thrombus formation at sites of injury in blood vessels. GT is classified into 3 subtypes, depending

(c) The Author(s). 2019 Open Access This article is distributed under the terms of the Creative Commons Attribution 4.0 International License (http://creativecommons.org/licenses/by/4.0/), which permits unrestricted use, distribution, and reproduction in any medium, provided you give appropriate credit to the original author(s) and the source, provide a link to the Creative Commons license, and indicate if changes were made. The Creative Commons Public Domain Dedication waiver (http://creativecommons.org/publicdomain/zero/1.0/) applies to the data made available in this article, unless otherwise stated. 
on the level of $\alpha \operatorname{IIb} \beta 3$. Affected individuals show mucocutaneous bleeding with the absent of platelet aggregation in response to all physiologic stimuli. They show normal platelet count and morphology. In addition, platelet $\alpha \operatorname{IIb} \beta 3$ deficiency or dysfunction should always be confirmed.

Laboratory tests in severe GT show no platelet aggregation in response to all physiologic agonists and show reduced or absent clot reaction [5]. Patients with type I or classical GT have a virtual absence of $\alpha \operatorname{IIb} \beta 3$ complex $(<5 \%$ of normal). In type II GT, patients have up to $25 \%$ of the normal level of the complex. In the variant type, $\alpha \operatorname{Ilb} \beta 3$ level is near normal but functionally impaired, leading to defective binding of fibrinogen $[6,7]$.

The $\alpha \operatorname{IIb} \beta 3$ complex integrin is encoded by ITGA2B and ITGB3 genes (GPIIb and GPIIIa respectively). In brief, integrin synthesis occurs in the megakaryocytes with the $\alpha \operatorname{IIb} \beta 3$ complex formation in the endoplasmic reticulum (ER). The pre-GPIIb-IIIa complex is then transported to the Golgi apparatus, where sugar modification and cleavage of GPIIb into a heavy $(M, 125,000)$ and light chain $(M, 23,000)$ takes place [5]. GPIIb heavy and light chains remain covalently linked by a single disulfide bond. Following these processing events within the Golgi, the mature GPIIb-IIIa complex is then transported to the cell surface, where it exists in a resting state awaiting cellular activation. Noncomplexed or incorrectly folded gene products fail to undergo processing in the Golgi apparatus and are rapidly degraded intra-cellularly.

A continually updated mutation database is available on the sinaicentral.mssm.edu/intranet/research/glanzmann website $[3,4]$. The molecular and functional characterization of the mutations has provided important insights to the better understanding of the biosynthesis and structure-function relationships of the $\alpha \operatorname{IIb} \beta 3$ complex with the disease. It also adds important knowledge about the biology of other molecules of the integrin family $[8,9]$.

Genetic testing in GT patients is very important in the quality of life of the affected individuals and it facilitates prenatal diagnosis (PND) or pre-implantation genetic diagnosis (PGD) for at-risk families. The identified mutations will span the current database of the mutations of GT.

We aimed at identifying mutations underlying GT in Iranian patients from different parts of Iran. Most of the documented mutations were single nucleotide substitutions but large deletions are rarely reported. This study is reporting a novel large deletion of $367 \mathrm{bp}$ in ITGA2B gene encompassing the last part of the first intron and the beginning part of the second exon of the gene.

\section{Material and methods}

\section{Patients' description}

Sixteen unrelated families with the diagnosis of GT were investigated for the ITGA2B and the ITGB3 genes mutations. The patients were referred to Medical Genetics Laboratory of Dr. Zeinali by hematologists. They were referred from the different parts of the country covering different races and ethnicities. Diagnosis of GT was based on the clinical picture of recurrent nasal hemorrhage, purpura, prolonged bleeding time (BT) and normal platelet count. Clinical features of each patient are reported in Table 1. Kawsar Human Genetics Research Center (KHGRC) ethical committee approved the research proposal.

Genetic counseling was performed and all participants signed informed consent forms. $10 \mathrm{ml}$ of peripheral blood of the patients and their parents were provided in tubes containing EDTA as an anticoagulant. Genomic DNA were extracted by salting out method [10].

\section{Molecular genetic study}

Genetic study of the patients was conducted using direct sequencing of the entire coding and exon-intron boundaries of the ITGA2B and the ITGB3 genes [3, 4]. Primer sequences are available upon request. PCR amplification was performed in a total volume of $25 \mu$ l containing $2.5 \mu \mathrm{l}$ of 10X buffer (Kawsar Biotech Co., Tehran, Iran, KBC), $2.5 \mu \mathrm{l}$ DMSO (Sigma-Aldrich, USA), $0.96 \mathrm{mM}$ dNTPs (KBC), $4 \mathrm{mM} \mathrm{MgCl} 2$ (KBC), 6 pmol of forward and reverse primers, $1 \mathrm{U}$ Taq polymerase $(\mathrm{KBC})$, and 30 ng of genomic DNA as a template. The thermocycling consisted of 1 cycle of initial denaturation at $95^{\circ} \mathrm{C}$ for 5 min, followed by 30 cycles of 1 min denaturation at $95^{\circ}$ C, 1 min annealing at $64^{\circ} \mathrm{C}$, and $1 \mathrm{~min}$ extension at $72^{\circ}$ $\mathrm{C}$, following a final extension at $72{ }^{\circ} \mathrm{C}$ for $10 \mathrm{~min}$ and storing the PCR product in $4{ }^{\circ} \mathrm{C}$.

Sequencing.

PCR products were directly sequenced using BigDye Terminator kit (Thermo Fisher Scientific, USA, TF) according to the manufacturer's protocol. Then the samples were run on an ABI3130XL Genetic Analyzer (TF). Sequences were compared with human genomic and cDNA sequences of the genes with the accession numbers of ITGA2B (NM: 000419, NC:000017) and ITGB3 (NM:000212, NC:000017). Mutations nomenclature was conducted according to the recommendations of Human Genome Variation Society [11].

\section{Characterization of a deletion breakpoint}

Systematic failure to amplify the 2nd and 3rd exons (since these exons are amplified together) of the ITGA2B suggested deletion of these two exons. To exactly determine the breakpoint, PCR- amplification with forward (F) of 2nd exon (2-3) and reverse (R) of 4th exon (3-4) was carried out. Primer sequences are listed below:

ITGA2B (2-3) F: GTCTGTGAGGTGTCATTGAGGA. ITGA2B (3-4) R: GCTTCACAGTAACGCTTGTCC. 


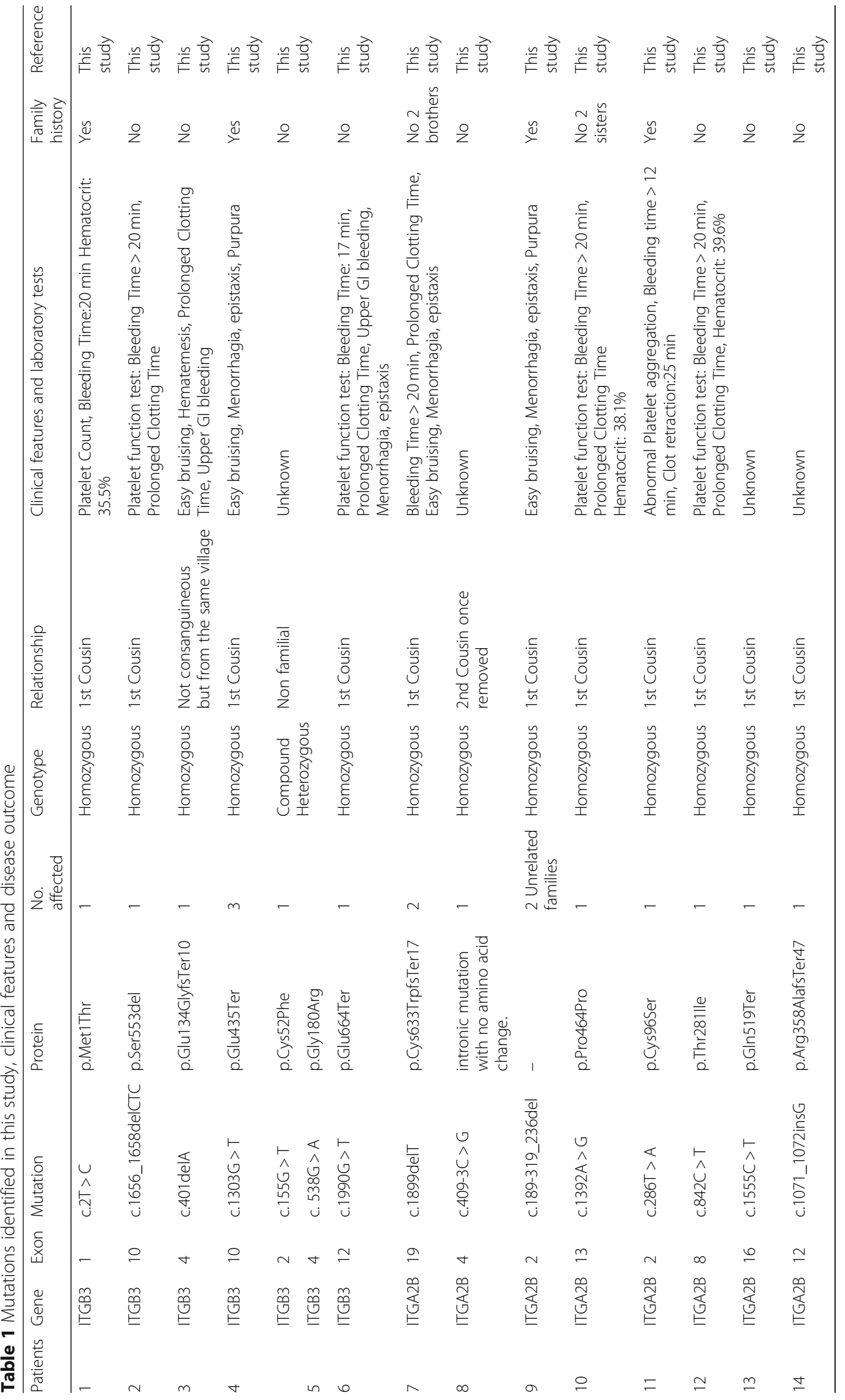


To find out the break point of this deletion, primer walking and subsequent sequencing were applied. The reaction mixture and PCR parameters were as above.

In order to check the frequency of novel missense variants, we performed ARMS-PCR in 100 ethnically matched healthy controls. Sequences of ARMS primers for these tests can be provided upon request. Bioinformatics analysis was also performed to elucidate the clinical significance of the identified variants. We used available online bioinformatics tools such as SIFT and PolyPhen Project to see the impact of missense variants on disease pathogenicity. Also, Mutation Taster was used for deletion and insertion variants [12].

\section{Results}

The study included sixteen unrelated families with the diagnosis of GT. As it was expected most of the patients were the result of consanguineous marriages (14/16 families, $87 \%)$. Sequencing analysis revealed totally 15 different mutations in the ITGB3 and the ITGA2B genes (Table 1). All identified mutations were homozygous except one in the ITGB3, which was in the form of compound heterozygous. Mutations in the ITGB3 gene consist 7 different mutations including 4 missense mutations [c.2T > C, c. $155 \mathrm{G}>$ T, c. $538 \mathrm{G}>\mathrm{A}$, c. 1990 $\mathrm{G}>\mathrm{T}$ ], a nonsense mutation [c.1303 $\mathrm{G}>\mathrm{T}$ ], one small 3 nt deletion [c.1656_1658delCTC] and a deletion of a single nt [c.401delA]. Interestingly all identified mutations in this gene were novel (Table 1).

Mutation scanning in the ITGA2B revealed 8 different mutations including an insertion [c.1071_1072insG], 2 missense mutations [c.286 T > A, c.842 C > T], a nonsense mutation [c.1555 C>T], 2 deletions [c.189-319_236del, c.1899 del T], one splice site mutation [c.409-3 C > $\mathrm{G}]$ and one synonymous mutation that might alter the normal splicing process [c.1392 A > G].

The identified variants were heterozygote in parents and were not present as homozygote in their healthy members of the family as expected in cases where mutations are deleterious. To further confirm the pathogenicity of the missense variants, we checked the mutation in 100 random people and they were normal which supports the pathogenicity of the novel variants.

In a family, while sequencing exons 2 and 3, we could not get any band in the patients suggesting that theses exons may be deleted. In order to characterize the deletion breakpoints, primer walking and sequencing were performed. While the size of DNA fragment in normal cases was nearly $1500 \mathrm{bp}$ as expected but the size of DNA product from the patient was around $1200 \mathrm{bp}$. Subsequent sequencing of this fragment confirmed deletion of $367 \mathrm{bp}$ covering $319 \mathrm{nt}$ from intron 1 and $48 \mathrm{nt}$ in the second exon (Fig. 1).

\section{Discussion}

GT is a rare congenital bleeding syndrome, which results in deficient platelet aggregation due to the absence or dysfunction of the fibrinogen receptor $\alpha \operatorname{IIb} \beta 3$ [12]. However, GT is rare worldwide but is relatively frequent in inbred populations such as Iraqi-Jews, Indians, and Arabs in Jordan, Israel, and Saudi Arabia. The exact number of GT patients in Iran has not been clearly reported. But it seems that the disease incidence will be higher from the estimated 1 in 200,000 in the US population. Because Iran is a country with nearly $38.4 \%$ rate of consanguineous marriage and at the other hand autosomal recessive mode of inheritance of the disease will add to the number of patients [13]. To the best of our knowledge, this study is the first comprehensive study on the molecular aspects of GT in Iranian population. Needless to say there is another similar study carried out by Kazemi et al. [14] on Iranian population but only three deleterious mutations have been reported out of 20 families affected with GT. However, none of the mutations reported by them is repeated in our study.

In Table 1 we have provided types of mutations and their effects at the amino acid level. Further in silico analysis was performed (Table 2) to find how these changes can be pathogenic. Obvious ones will not be discussed.

The first patient (Table 1) with c. $2 \mathrm{~T}>\mathrm{C}$ point mutation which is located at the very beginning of the first exon of the ITGB3 gene ( $\beta 3$ subunit,) will change the methionine to threonine. Initiator methionine is very important for the initiation of protein synthesis.

Regarding the third patient with c.401delA mutation in the ITGB3, and based on mutation taster prediction [15] it is disease causing change. The fourth patient with c.1303G > T [p. Glu435Ter] mutation, causes change of glutamic acid to a termination codon leading to premature stop codon with only 434 residues which most probable will not function properly. The other patient had c. $155 \mathrm{G}>\mathrm{T}$ mutation in the ITGB3 gene, changing cysteine into phenylalanine. The wild-type residue is involved in a cysteine bridge formation, which is important for the stability of the protein. Only cysteine can make these types of bonds, so the mutation causes loss of this interaction and will have a severe effect on the $3 \mathrm{D}$-structure of the protein. Together with the loss of the cysteine bond, the differences between old and new residue can cause destabilization of the structure [16], This prediction is also supported by SIFT and Polyphen results. The c. 538 G > A [p. Gly180Arg] mutation in the ITGB3 changes glycine into arginine and introduces an amino acid with different properties that can disturb this domain and abolish its function. Because the wild-type residue, glycine, is the most flexible of all residues. 
AGAAAAGACTTCCTGTGGAGGAATCTGAAGGGAAGGAGGAGGAGCTG GCCCATTCCTGCCTGGGAGGTTGTGGAAGAAGGAAGATGGCCAGAGCT TTGTGTCCACTGCAAGCCCTCTGGCTTCTGGAGTGGGTGCTGCTGCTCT TGGGACCTTGTGCTGCCCCTCCAGCCTGGCCCTTGAACCTGGACCCAGT GCAGCTCACCTTCTATGCAGGCCCCAATG CAGCCAGTTTGGATTTTCA CTGGACTTCCACAAGGACAGCCATGGGAGGTGAGCCGTAAGGGAAGTT

2725 bp intronic region was missed out to show the changes

AAAACCAGCTCTCAAGAGGGGATCTGGTAACAGTCTAGGCAG ICATTC CAGGGAGCATGTGAACCGCTGGTTCTTGTTGCGGGTGGAAGGAtggaggtgt tgtacagagtttaggtctttttcagcaaagatctcaaacccetggtgttcaaatcaaac caaatggttatagtcc cagctct actctcaactcactgggttactttagc cacgtgattgc c ctcgctgagagtcggtttcactgtc cataagatga agaagta catcacggtggtctgtgaggtgtcattgaggaaagatggtccagtgcccccatgc cacatggecttccgg, yagtgct cccagc gecggegccagggectgggatacgctggaatctgc cggcgetcacccagcttcctatgcaga atcgtggtgggegeccegcggaccetgggecccagc caggaGGAGACGGGCGGCGTGTTC두 GTGCCCCTGGAGGGCCGAGGGCGGCCAGTGCCCCTCGCTGCTC ГTTGAC CTCCGTGAGTCCCAGGCAAGGAGAGCAAGGTTGGGGTCAGAG GACGT GGACTGCCGGGCTTCAGCGCCCCACCCCTTCTTGTGCCTTCCAGGTGAT GAGACCCGAAATGTAGGCTCCCAAACTTTACAAACCTTCAAGGCCCGC CAAGGACTGGGGGCGTCGGTCGTCAGCTGGAGCGACGTCATTGTGGTG GGCCCCGCGGTACAGGGCACAGGGAACAATCGGGGGCAGGGACACCT

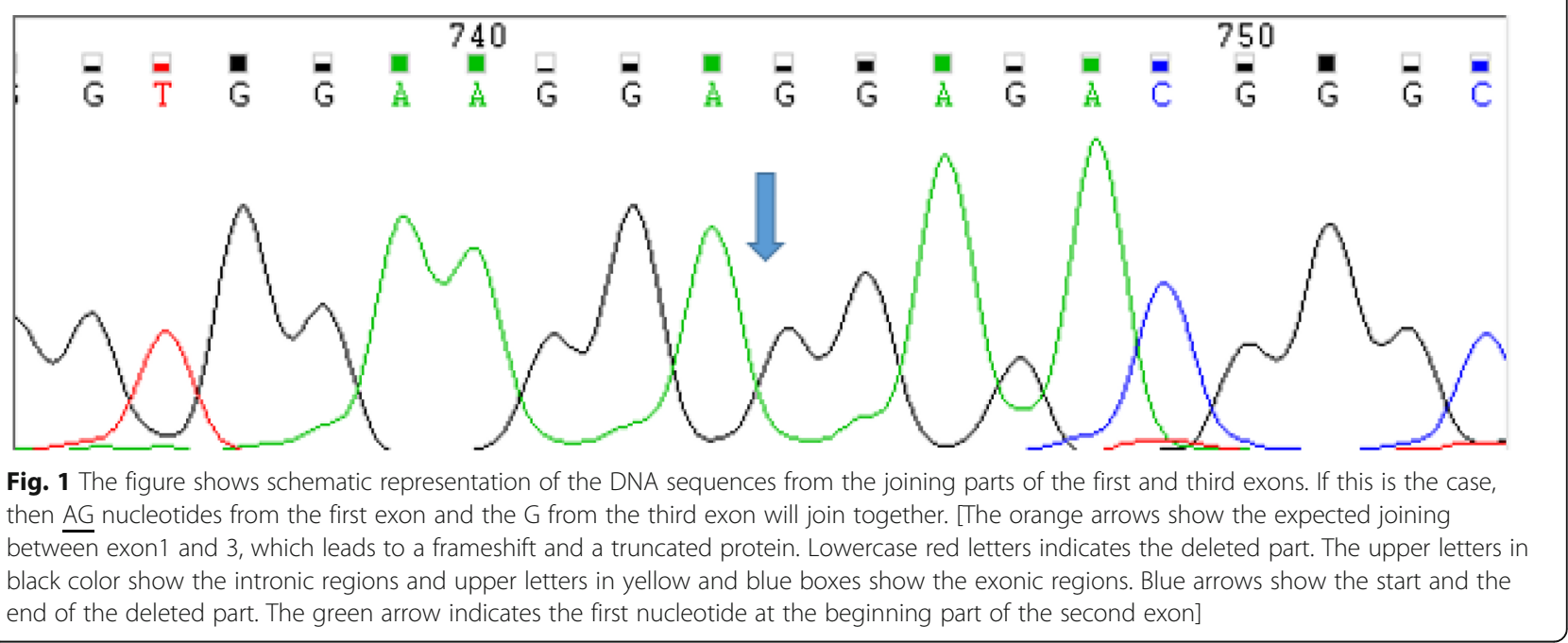

Mutation of this glycine can abolish this function. This flexibility might be necessary for the protein's function and by this mutation the torsion angles for this residue are unusual [16]. This prediction is also compatible with the SIFT and Polyphen prediction (Table 2).
The deletion of $\mathrm{T}$ nucleotide in exon 19 of ITGA2B gene (i.e. c.1899 del T [p. Cys633TrpfsTer17]) causes a frameshift in protein structure. HSF (Human splice finder) predicted that this frameshift created an exonic ESS site that has a potential to alter the splicing [17]. Regarding c.409-3 C > G 


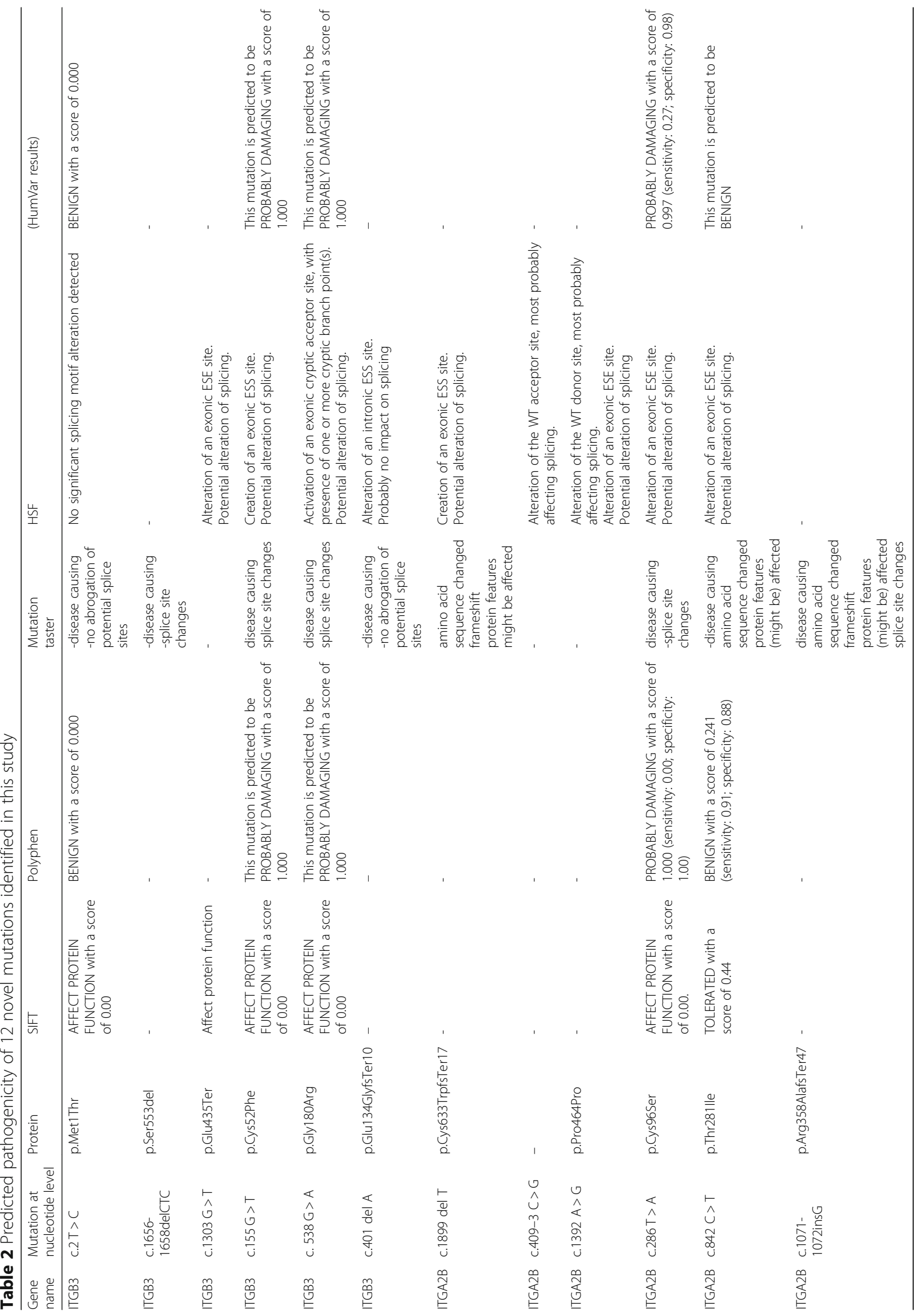


and c.1392 A > G mutations in the ITGA2B which occurred near the potential splice sites of the protein, this can alter the wild type acceptor and donor site respectively. The c.189-319_236del mutation at ITGA2B gene leads to the elimination later part of the intron one and the beginning part of second exon. This deletion, therefore, deletes the splice acceptor site. This in turn should cause deletion of the entire exon 2 because there is no alternative splice site within the remaining part of exon 2 and therefore the remaining part of this exon will be eliminated via splicing of intron 1 (donor site), joining of exon 1 and exon3. Therefore it is expected that through this splicing the exon 1 will be joined with exon 3 . Figure 1 shows that if this would be the case then amino acids 63rd to 103rd will be lost and a frameshift would be resulted. The frameshift mutation leads to a truncated protein which loss of function would be resulted. Since we could not obtain fresh blood from the patient to perform RNA analysis, the above conclusion can be provided. However, whatever the consequence at the molecular level, we can claim that resulting effect at the protein level is having damaging effect on the patient.

It should be added that the identified variants were not present in the single nucleotide polymorphism database (Exome Variant Server, http://evs.gs.washington. edu/EVS/).

Regarding phenotype-genotype correlation, more studies with larger sample size is recommended.

It is interesting to see so many novel mutations in a rather small number of families (14/16) indicating several independent founder mutations. If we combine other mutations reported by others (Kazemi et al.) with ours it might become even more interesting. This may mean that either these genes are highly mutable (which may not be true since the disease is not so prevalent), or Iranian population is so diverse. This diversity has many historical proofs, which are beyond the scope of this paper but it worth mentioning.

\section{Conclusions}

In conclusion, our investigation of 16 patients, and in some cases including their families, revealed a number of different mutations within the $\alpha I I b$ and $\beta 3$ subunits associated with causing GT. This vast number of different mutations in different provinces of Iran indicates the genetic heterogeneity in the studied group as well as in GT in general. Further investigation on the prevalence of each mutation from each ethnicity may reveal different picture.

\section{Abbreviations}

bp: Base pair; BT: Bleeding time; del: Deletion;

EDTA: Ethylenediaminetetraacetic acid; ER: Endoplasmic reticulum; GP: Glycoprotein; GT: Glanzmann thrombasthenia; ins: Insertion; KHGRC: Kawsar Human Genetics Research Center; Nt: Nucleotide; PCR: Polymerase chain reaction; PGD: Pre-implantation genetic diagnosis; PND: Prenatal diagnosis; U: Unit; ng: nanogram

\section{Acknowledgements}

We gratefully thank Dr. Zeinali Medical Genetics Lab staff for helping us to perform the molecular genetic testing on their patients. The authors extend their gratitude to the patients and their willingness to participate in this study.

\section{Funding}

This project was supported by a grant from the Kawsar Human Genetics Research Center.

\section{Availability of data and materials}

The datasets are available from the corresponding author on reasonable request.

\section{Authors' contributions}

ZMF was involved in acquisition, analysis and interpretation of the data and drafting the manuscript. FMS was involved in interpretation of the data and in drafting the manuscript. GS was involved in in-silico analysis of datasets, interpretation of the data and in drafting the manuscript. BH was involved in patients counseling and data collection, analysis and interpretation of the data and in drafting the manuscript. ST was involved in mutation analysis, interpretation of the data and in drafting the manuscript. DS was involved in primer designing, interpretation of the data and in drafting the manuscript. $\mathrm{JM}$ is involved in molecular testing and interpretation of the data. SZ is involved in interpretation of the data and in drafting the manuscript. AM is involved in interpretation of the data and in drafting the manuscript. ZS was involved in interpretation of the data and in drafting and finalizing the manuscript. All authors have read and approved the final manuscript.

\section{Ethics approval and consent to participate}

The study questionnaire and consent forms have been attained from the participants and all participants signed informed consent forms.

The Iranian Hemophilia Society approved the protocol.

Consent for publication

Authors declare that there is no conflict of interest.

\section{Competing interests}

The authors declare that they have no competing interests.

\section{Publisher's Note}

Springer Nature remains neutral with regard to jurisdictional claims in published maps and institutional affiliations.

\section{Author details}

'Dr. Zeinali's Medical Genetics Lab, Kawsar Human Genetics Research Center, No. 41 Majlesi St., Vali Asr St, Tehran 1595645513, Iran. ${ }^{2}$ Department of Biology, Pardis International, University of Guilan, Rasht, Iran. ${ }^{3}$ Department of Medical Genetics and Molecular Biology, Faculty of Medicine, Iran University of Medical Sciences, Tehran, Iran. ${ }^{4}$ Department of Molecular Medicine, Biotechnology Research Center, Pasteur Institute of Iran, Tehran, Iran.

Received: 4 January 2018 Accepted: 5 March 2019

Published online: 27 April 2019

\section{References}

1. Poon MC, D'Oiron R, Von Depka M, Khair K, Negrier C, Karafoulidou A, HuthKuehne A, Morfini M. International data collection on recombinant factor V, congenital platelet disorders study G: prophylactic and therapeutic recombinant factor VIla administration to patients with Glanzmann's thrombasthenia: results of an international survey. J Thromb Haemost. 2004; 2:1096-103.

2. Handin RI. Inherited platelet disorders. Hematology Am Soc Hematol Educ Program. 2005;1:396-402.

3. Pillitteri D, Pilgrimm AK, Kirchmaier CM. Novel mutations in the GPIIb and GPIlla genes in Glanzmann Thrombasthenia. Transfus Med Hemother. 2010; 37(5):268-77.

4. Rosenberg N, Hauschner H, Peretz H, Mor-Cohen R, Landau M, Shenkman B, Kenet G, Coller BS, Awidi AA, Seligsohn U. A 13-bp deletion in alpha (IIb) gene is a founder mutation that predominates in Palestinian-Arab patients with Glanzmann thrombasthenia. J Thromb Haemost. 2005;3:2764-72. 
5. Wilcox DA, Wautier JL, Pidard D, Newman PJ. A single amino acid substitution flanking the fourth calcium binding domain of alpha IIb prevents maturation of the alpha IIb beta 3 integrin complex. J Biol Chem. 1994:269:4450-7.

6. Carl Maximilian Kirchmaier DP. Diagnosis and Management of Inherited Platelet Disorders. Transfus Med Hemother. 2010;37(5):237-46.

7. George JN, Caen JP, Nurden AT. Glanzmann's thrombasthenia: the spectrum of clinical disease. Blood. 1990;75:1383-95.

8. Bellucci S, Caen J. Molecular basis of Glanzmann's Thrombasthenia and current strategies in treatment. Blood Rev. 2002;16:193-202.

9. Phillips DR, Charo IF, Parise LV, Fitzgerald LA. The platelet membrane glycoprotein Ilb-Illa complex. Blood. 1988;71:831-43.

10. Miller SADD, Polesky HF. A simple salting out procedure for extracting DNA from human nucleated cells. Nucleic Acids Res. 1988;16(3):1215.

11. den Dunnen JT, Dalgleish R, Maglott DR, Hart RK, Greenblatt MS, McGowanJordan J, Roux AF, Smith T, Antonarakis SE, Taschner PE. HGVS recommendations for the description of sequence variants: 2016 update. Hum Mutat. 2016;37(6):564-9.

12. Schwarz JM, Rödelsperger C, Schuelke M, Seelow D. MutationTaster evaluates disease-causing potential of sequence alterations. Nat Methods. 2010;7:575-6.

13. Saadat M, Ansari-Lari M, Farhud D. Short report consanquineous marriage in Iran. Ann Hum Biol. 2004;31:263-9.

14. Kazemi A, Abolghasemi H, Kazemzadeh S, Vahidi R, Faranoush M, Farsinejad A, Ala F. Molecular characterization of Glanzmann's thrombasthenia in Iran: identification of three novel mutations. Blood Coaqul Fibrinolysis. 2017:28:681-6.

15. Adzhubei IA, Schmidt S, Peshkin L, Ramensky VE, Gerasimova A, Bork P, Kondrashov AS, Sunyaev SR. A method and server for predicting damaging missense mutations. Nat Methods. 2010;7:248-9.

16. Venselaar H, te Beek TA, Kuipers RK, Hekkelman ML, Vriend G. Protein structure analysis of mutations causing inheritable diseases. An e-science approach with life scientist friendly interfaces. BMC Bioinf. 2010;11:548.

17. Desmet F-O, Hamroun D, Lalande M, Collod-Béroud G, Claustres M, Béroud C. Human splicing finder: an online bioinformatics tool to predict splicing signals. Nucleic Acids Res. 2009;37:e67.

Ready to submit your research? Choose BMC and benefit from:

- fast, convenient online submission

- thorough peer review by experienced researchers in your field

- rapid publication on acceptance

- support for research data, including large and complex data types

- gold Open Access which fosters wider collaboration and increased citations

- maximum visibility for your research: over $100 \mathrm{M}$ website views per year

At $\mathrm{BMC}$, research is always in progress.

Learn more biomedcentral.com/submissions 